\title{
Conceptual Security Framework based on Cloud Computing Services for Spatial Database
}

\author{
Mazloh Al-Enazi \\ Arab Academy for Science, \\ Technology and \\ Maritime Transport \\ Alexandria, Egypt
}

\author{
Saleh Mesbah \\ Arab Academy for Science, \\ Technology and \\ Maritime Transport \\ Alexandria, Egypt
}

\author{
Amani Anwar \\ Arab Academy for Science, \\ Technology and \\ Maritime Transport \\ Alexandria, Egypt
}

\begin{abstract}
Cloud Computing is a point of attraction and got a lot of attention because of the numerous abilities it offers. On the other hand spatial-data is vital and considered to be a global important aspect. The security challenges it poses online is significant. The cloud computing security solution is used to avoid all the risks or the potential vulnerabilities being introduced by the computer industry. Solution is moving data to the cloud, while it is imperative for cloud service providers guarantying the security of geo-spatial-data information, providing cloud service users the terms and conditions of services provided by the cloud service providers and trusting them with their data. Although many different security solutions are provided to secure the data within the enterprise, the cloud solution is becoming vital for many other reasons too. The paper proposes a conceptual spatial data based on cloud infrastructure and applied security methods framework, which is capable of achieving user authentication and authorization.
\end{abstract}

\section{Keywords}

Spatial database, security, Cloud, Computing

\section{INTRODUCTION}

The enormous advancements and developments of spatial data and cloud computing technology have been rising recently, especially the cloud computing technology and that's because of them any advantages provided i.e. data storage, data viewing and data processing. Moreover, cloud provides the functionality in replacing computers in a very short time making the whole system elastic and function determinant [1].

The cloud computing technology provides the whole world with huge data processing, storage and maintaining facilities providing the spatial data seekers to rely on to retrieve geospatial-data based on cloud computing infrastructure over the global World Wide Web [2].

On the other hand, cloud computing has some issues as any new technological trend, and the issues are concerned with security, privacy and authentication, authorization, access control problems. Recently a brand new connection happened when Cloud computing and GIS or geo spatial data that is called spatial cloud computing. When it comes to spatial cloud computing, the end-users have defined accurate authentication, and authorization access to the only when applying the cloud security, thus spatial data will be retrieved online without the need of stationary devices [3].

\section{LITERATURE REVIEW}

With the advancement of Earth perception innovation and GIS innovation, enormous geospatial-data, particularly remote detecting information, are amassing and being utilized as a part of different commercial ventures and research organizations. Geospatial-data are more mind boggling than other information sorts since they contain area data. Moreover, on the grounds that the volumes of geospatial-data are tremendous, a lot of space and time utilization of remote detecting pictures is required for some applications, particularly in the field of natural sciences.

For instance, researches on worldwide environmental change requires geospatial-data covering more than individual nations or landmasses. It is troublesome for people or scientists not just to get every one of the information they require additionally to handle the gigantic measure of geospatial-data in view of an absence of superior computational foundation. Step by step instructions to successfully coordinate the persistently developing geospatial-data assets and give people in general administrations, for example, spatial-data downloading, putting away, and breaking down has turned into a critical issue [4].

Analysts at home and abroad have a now concentrated how to apply distributed computing in enormous geospatial-data administration and sharing administrations. Scientists had a go at consolidating distributed computing with spatial-data stockpiling and preparing and tackled two key issues by utilizing Map Reduce structure, a R-Tree record for a wide range of Spatial-data and remote detecting picture quality assessments. Blower (2010) [5] executed a web map administration on Google App Engine and portrayed the challenges in creating GIS frameworks on people in general cloud. Siládi, Huraj, Polčák, and Vesel (2012) connected distributed computing in GIS science via doing Spatial-data introducing on the Amazon Elastic Compute Cloud [6].

This was vital, as a matter of fact geospatial-data procedures are considered exceptionally tedious and information serious, particularly for huge and complex datasets. Rezgui, Malik, and Yang (2013) additionally utilized spatial interjection to survey the advantages of distributed computing for geosciences applications [3].

Researchers in literature reviews proposed an interoperable Spatial-data object model and re-planned spatial indexing calculations, for example, Quad-Tree and R-Tree, to fathom the downsides of Spatial-data stockpiling in the normal distributed computing stage. Others broke down the need of embracing distributed computing in the remote detecting information handling administration. What's more, by concentrating on the structure and key advancements of distributed computing, they actualized a model framework named Open RS-Cloud [7].

Also researchers in literature proposed a hypothetical distributed computing system to successfully prepare land 
asset administrations, in light of standard hypothesis and key innovations of distributed computing. Others contemplated key advances of distributed computing and executed an electronic graph cloud benefit that accomplished proficient administration of worldwide electronic outline information and gave customized, adaptable, and very accessible administration [8].

Every one of these works has advanced change of the hypothetical investigation of the mix of distributed computing with enormous geospatial-data administration. Be that as it may, a few issues still exist. To start with, the frameworks created are for the most part flimsy models typically missing point by point portrayals and with minimal substance that merits referencing. Second, the model frameworks executed were tried with a little measure of information, insufficient to approve the execution of a cloud-based framework. Third, numerous studies utilized an open cloud stage, for example, Google, Amazon, Microsoft, and so on to build up their cloud based geospatial-data stage.

\section{PROPOSED CONCEPTUAL FRAMEWORK}

In the most recent couple of years, geospatial information security has gotten to be one of the principle zones of exploration all around the world. Geo-spatial-data It is a gathering of extensive variety of apparatuses that catches, stores, examinations, oversees and uses huge volumes of geospatial information and conveys geo-spatial-data and administrations for huge simultaneous clients.

Along these lines, one of the necessities is the capacity to handle the gigantic volume of spatial information and guarantee the required execution with operational reliability.

\subsection{Proposed Framework}

The proposed framework has two main component layers where the security is applied for both. The first layer is considered to be the (internal layer), which is mainly the storage layer that is concerned with very accurate and reliable access control of the geospatial database. This layer is an Authentication that provides transferring of the private Geospatial-data to the main own servers with the aid of trust and privacy management protocols. The second layer (outer layer) which is considered to be a distribution layer works with the end-users connection between themselves and GIS web services or what is mentioned to be trusted servers to retrieve geospatial data. In other words the user will need to connect to web services / trusted servers, and then the mutual authentication process through cloud services is a must providing a secure system from malicious attacks by unauthorized users. The system architecture of the Geospatial Data Cloud is as shown in Figure 1.

Furthermore, the Data Processing layer which is composed of the data processing and all the processes regarding the virtual machines those are on cloud. As shown the Figure 2 the framework stages the conceptual idea of Spatial-data secured on Cloud Environment.

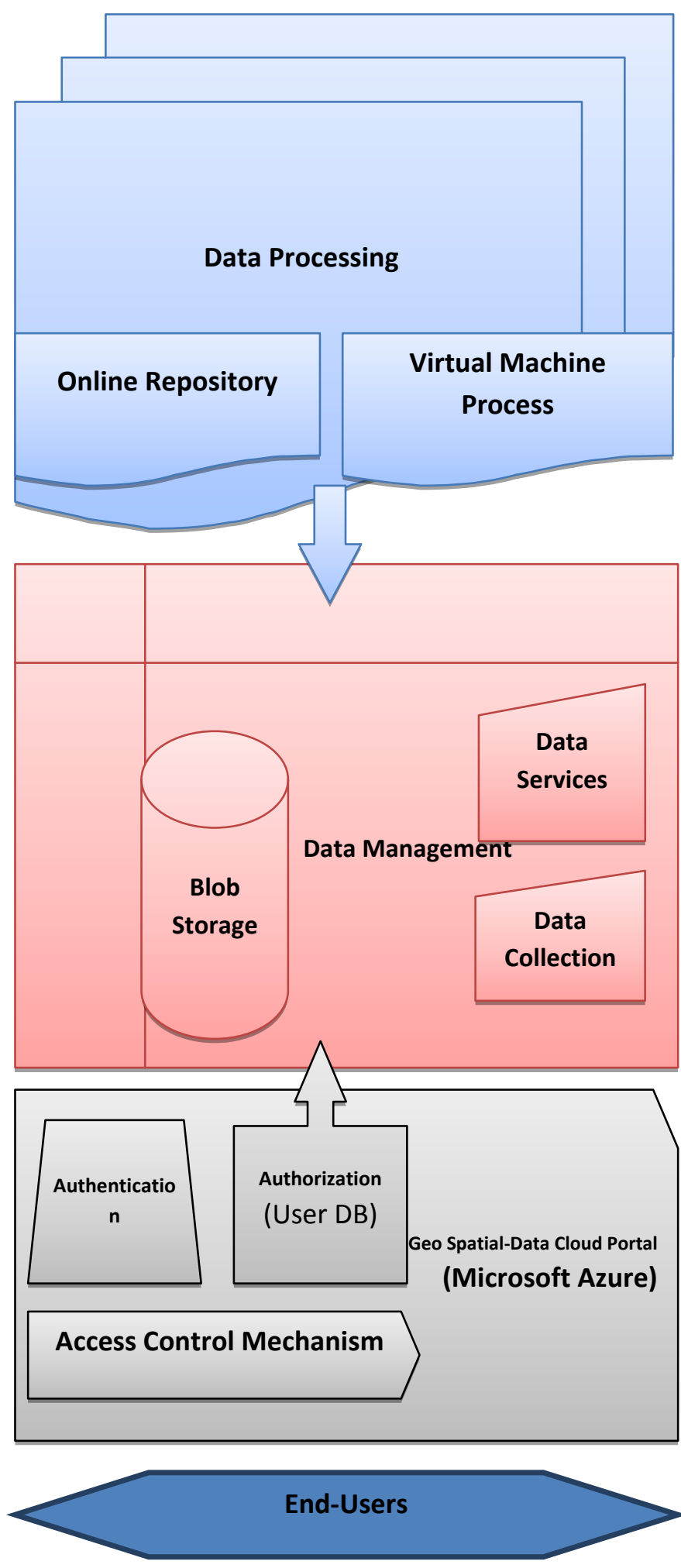

Figure 1: Proposed system architecture of the Geospatial Data Cloud 


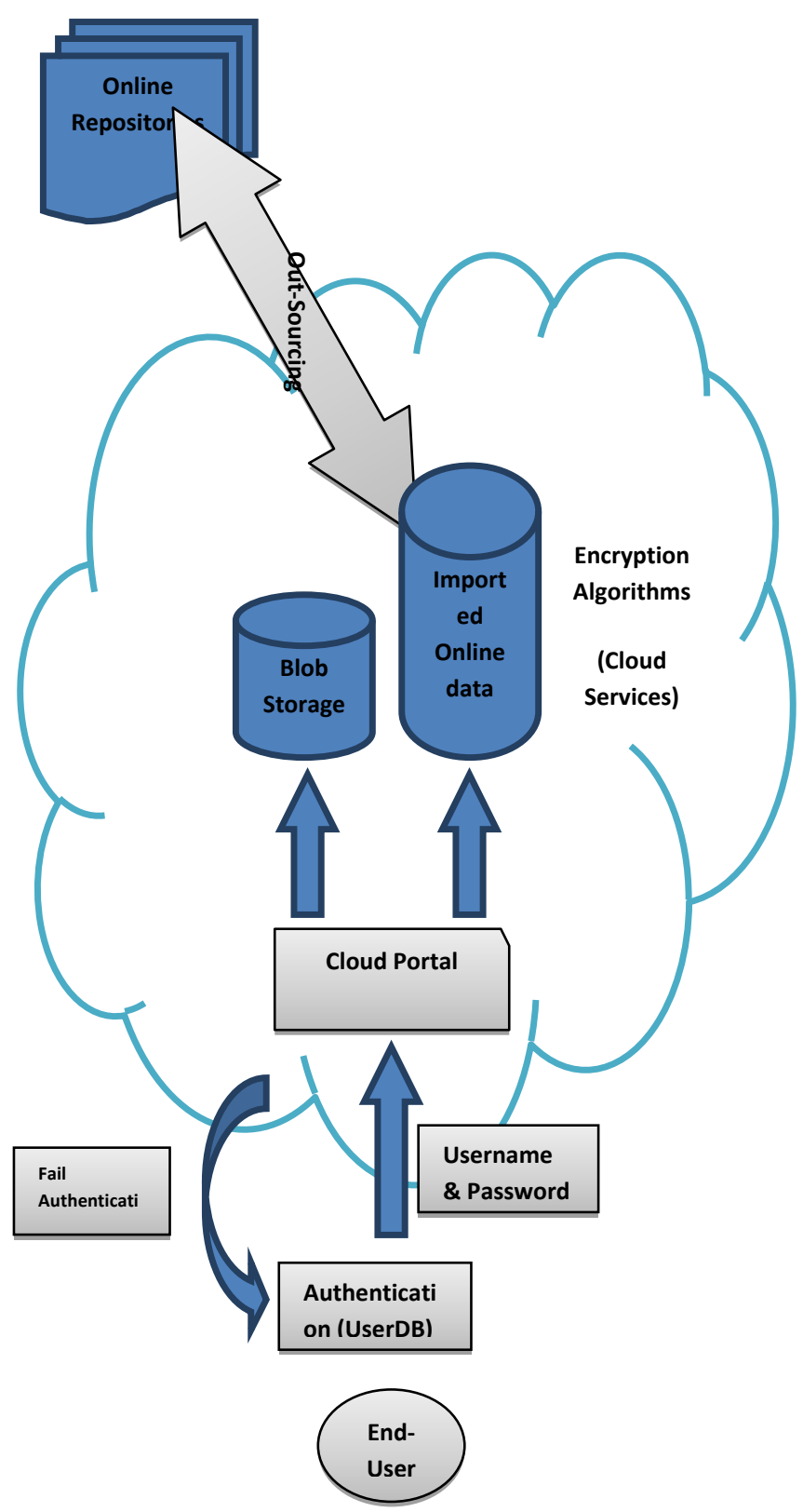

Figure 2: The proposed Framework

\subsection{Geospatial Data Cloud Portal}

On top of the architecture of the Geospatial Data Cloud Portal is a one-stop entrance that gives an assortment of administrations through a program. Through this layer, any approved client can utilize arranged terminal gear to login to the cloud stage as per a standard open application interface to appreciate cloud administration from anyplace. Every one of the client's needs to do is submit demands. The principle elements of the entry are: information recovery, group information downloading, remote detecting picture perception, private space to store information, etc.

\subsubsection{Application Layer}

The geospatial application the composite layer and is the main component of the Geospatial Data Cloud. This layer contains two modules, the cloud based data management and the cloud based data processing. The two modules work not only to increase the performance using cloud computing technology resources but also connect cloud computing with GIS technology.
Virtual technologies that enable cloud architecture perform scalability. As a matter of fact, cloud computing considered to be virtualized resource that provides storage and computing resources. In the Geospatial Data Cloud, a distributed file system is used in order to store remote sensing data. A virtual server virtualizes the Post-Gresql cluster and enables users to access all the underlying resources by way of the cloud host. Also, by scheduling tasks, it can locate all the nodes to support the execution of a given programming model.

\subsection{Data Management Platform}

This layer, data management layer have two important modules, the data collection, which includes automatic metadata crawling data from the online resources. Second is the data service, which provides huge Geo-spatial-data storage in Blob.

\subsection{1 data collection}

Information is the premise of a cloud administration stage. In the field of remote detecting, the wellsprings of the information, for instance, LANDSAT, MODIS, EO-1, DEM, NCAR, NOAA, LUCC, and so on, are various. As information substances are regularly unpredictable and heterogeneous, metadata are frequently used to portray information elements. Keeping in mind the end goal to bind together the outflow of data and information elements, the researchers concentrated on information expression in geosciences, universal basic information representation, and information interoperability models.

At that point the researchers made a data display that are locally perfect. On the premise of these works, a nearby metadata converter was produced to parse, change, and concentrate data from metadata from various information assets.

First step, the process randomly login to the FTP website and retrieves the metadata file. Secondly, it finds out if the same data already exist. If the data exist, then it reads the next file, if the data is not found, the module reads the whole contents and save the new data in a database. Finally, creates URLs, and publishes the data on cloud for retrieval by users.

\subsubsection{Data Service}

After the crawl step is finished and the data is collected from the online sources, the data services can sheared files and exports the data, then stores the data in Post greSQL and Post GIS through clustering technology. Finally, publishes the data into the server storage and file directory.

\subsection{Access Control Mechanism}

Access control policies that is responsible of data access regulations. The main point is to ensure that control that is granted access to data through specific credentials. When concerned the importance of geospatial-data databases, the specific roles of users and the hierarchy of spatial data. The authorization for the access control is composed of user is or role and Event which denotes if any action occurs to permit/deny access permission and the operation to be executed on spatial data.

Concerning the out-sourcing of spatial-data information, security turns into the primary concern. The essential necessities o secure information outsourcing is secrecy of the outsourced information, client and additionally information protection and accuracy of question results. To accomplish security, geospatial information ought not to be outsourced. Rather, it ought to be initially changed into some space 
utilizing change methods and afterward outsourced to outsider server.

While dealing with spatial-data at specific out-sourcing level, the spatial-data needs to be protected. On the other hand, if the data is not protected this will lead to illegal copy and distribution or even disturbing. It's well known that the use of digital watermarking for copyright protection of Spatial-data is considered to be a good solution to work with as it's very easy to locate legitimate owner of the data (user or server) in case of tampering. Moreover, cryptographic algorithms like identity based encryption, makes it more powerful against various attacks.

\section{CONCLUSION}

The geospatial data cloud gives clients huge scale, remote detecting information assets, versatile registering and capacity assets, and a fundamental base environment by utilizing a cloud administration. The modules that create the stage are approximately coupled. Therefore, it is anything but difficult to find disappointment focuses and discover bottle necks amid the framework's execution and in this way upkeep and streamlining of the framework are encouraged. Furthermore, numerous different parts of change have been made in the geospatial data cloud. In the first place, various and colossal information sources are accessible. Truth be told, there are more than 350TB information substances accommodated free. As the information recovery is quick, it is extremely helpful and efficient for analysts to secure information. Second, nine information preparing models are given. Along these lines, clients don't need to buy elite computational gear and introduce information preparing programming, for example, ENVI, which are expensive. At long last, every one of the administrations is allowed to all clients. These days, there are more than 70,000 clients of the geospatial data cloud.

The selection of cloud computing in the field of geo-spatialdata is certainly engaging. Be that as it may, more work still should be done to enhance this stage later on. To begin with, the way toward redoing models and figuring assignments should be more accommodating. Likewise, as an information cloud stage, the geo-spatial-data cloud ought to import increasingly amazing information to clients.

With the quick improvement of Internet and correspondence innovation, it turns out to be anything but difficult to duplicate or disperse the geo-spatial-data. In this way copyright insurance, validness, security, and spatial information source following have gotten to be essential issues. In this paper, researchers have displayed a theoretical structure to satisfy these necessities. The proposed system postures security at database, as well as at dispersion level by applying different security strategies.

\section{REFERENCES}

[1] Liu, C.Y. (2013) Research and Practice on Key Technologies of Electronic Chart Cloud Service, PLA Information Engineering University, Zhengzhou, Henan, China.

[2] Liu, Y., Guo, W., Jiang, W. S., \& Gong, J. Y. (2009) Research of remote sensing service based on cloud computing mode. Application Research of Computers 26(9), pp 3428-3431.

[3] Rezgui, A., Malik, Z., \& Yang, C. (2013) Highresolution spatial interpolation on cloud platforms. In Proceedings of the 28th Annual ACM Symposium on Applied Computing, pp 377-382.

[4] Fay Chang, Jeffrey Dean, Sanjay Ghemawat et al. BigTable: a distributed storage system for structured data [A]. Operating Systems Design and Implementation, 2006.

[5] Blower, J. D. (2010) GIS in the cloud: implementing a Web Map Service on Google App Engine. In Proceedings of the 1st International Conference and Exhibition on Computing for Geospatial Research \& Application, ACM, p 34.

[6] Siládi, V., Huraj, L., Polčák, N., \&Vesel, E. (2012) A parallel processing of spatial data interpolation on computing cloud.In Proceedings of the Fifth Balkan Conference in Informatics, ACM, pp 193-198.

[7] Ali, A.,Irum, S.,Kausar, F., Khan, F.A. A cluster-based key agreement scheme using keyed hashing for Body Area Networks.Multimedia Tools and Applications vol. 66, pp.201-214, 2013.

[8] Ali, A., Khan, F.A. Energy-efficient cluster-based security mechanism for intra-WBAN and inter-WBAN communications for healthcare applications. EURASIP Journal on Wireless Communications and Networking, vol. 2013, no. 1, pp. 216, 2013. 